(c) 2018 IEEE. Personal use of this material is permitted. Permission from IEEE must be obtained for all other uses, in any current or future media, including reprinting/republishing this material for advertising or promotional purposes, creating new collective works, for resale or redistribution to servers or lists, or reuse of any copyrighted component of this work in other works. 


\title{
Impact of Reactive Obstacle on Molecular Communication between Nanomachines
}

\author{
Muneer M. Al-Zu'bi, Student Member, IEEE, Ananda S. Mohan, Senior Member, IEEE, and \\ Steve S.H. Ling, Senior Member, IEEE \\ Center for Health Technologies (CHT), Faculty of Engineering and Information Technology (FEIT) \\ University of Technology Sydney (UTS), NSW, Australia. \\ E-mail: \{muneer.al-zubi, ananda.sanagavarapu, steve.ling\}@uts.edu.au
}

\begin{abstract}
Molecular communication is an emerging technology for communication between bio-nanomachines in an aqueous environment. In this paper, we examine the effect of a reactive obstacle, which is placed in the diffusive molecular communication channel, on the expected number of the received molecules at the receiver. We develop a particle-based simulator that can predict the number of the received molecules for both passive and absorptive receivers by considering the impact of the reactive obstacle within the communication channel. The impacts of the reaction probability and radius of the obstacle on the received signal are examined and compared with the case of absence of the obstacle. The results show significant impact for the obstacle on the received signal, particularly, for obstacle with high reaction probability and large size.
\end{abstract}

Keywords-molecular communication,diffusion, biological, nanoscale, nanomachines, reactive obstacle.

\section{INTRODUCTION}

Molecular communication is an emerging technique to model the interaction and communication at nanometer to micrometer scales highly suitable biological molecular environments [1]-[2]. It enables the communication between the bio-nanomachines and biological cells inside the body using chemical molecules such ions and proteins [2]-[3]. The bio-nanomachines (NMs) are tiny devices made of nano-tomicroscale components of biocompatible materials which perform simple functions such as sensing, computation, and actuation [2]. Nanonetwork is an interconnection of many nanomachines that communicate with each other in a cooperative manner [1].

Molecular communication is a promising alternative technology for electromagnetic communication at nano- and micro- scales due to its biocompatibility and low energy requirements [3]. Moreover, using EM communications at this scale will require very small antennas and thus extremely high frequencies which could lead to high attenuation and poor propagation inside the body. Among MC transport mechanisms, molecular communication via diffusion (MCvD) is the most simple transport method which does not require energy or communication infrastructure [2][4]. Therefore, it is appropriate for many biomedical applications such as targeted drug delivery, in-body area nanonetworks, and lab-on-chip systems [5]. In MCvD, the information molecules diffuse randomly according to Brownian dynamic which can be mathematically modelled

This work is supported by the UTS FEIT Blue sky Research Grant 2017. using Fick's diffusion equations [6, 7]. In this random motion, the molecules propagate by utilizing the thermal energy that is already present in the environment. Thus, no source of external energy is required for diffusion-based molecular communications.

In [8], the authors present a new physical end-to-end model for molecular communication by modeling the emission, diffusion, and reception processes. The normalized gain and delay are evaluated as a function of the input frequency and transmission range. An energy model for molecular communication via diffusion (MCvD) with optimization based on channel capacity and the data rate is developed in [9]. In [10]-[11], an optimum design model for a passive receiver in a diffusive molecular communication channel is addressed. It includes the flow, noise, and enzymes in the propagation environment to mitigate intersymbol interference (ISI). In [12], analytical expressions for communication metrics, viz., pulse delay, pulse amplitude and pulse width, are derived for MCvD system using amplitude and energy detection techniques while in [13], a closed-form expression is derived for error probability using these detection techniques. An analytical expression for fraction of absorbed molecules by an absorptive receiver in MCvD system is derived in [14]. In [15], an analytical model is presented for a reversible receiver in MCvD system and an analytical expression is derived for the expected number of adsorbed molecules. Recently, in [16], the impact of interfering absorber receiver on another in $\mathrm{MCvD}$ channel has been addressed. Effect of positions of interfering receiver and transmitter are examined. However, in [16], the interfering receiver is modelled as a full absorber, which represents an idealization or special case of the irreversible receiver (partially absorber).

In this paper, we investigate impact of a partially absorptive obstacle, e.g., a biological cell, located in the communication link between transmitting nanomachine (TN) and receiving nanomachine $(\mathrm{RN})$, on the received signal. The number of the received molecules at the target site $(\mathrm{RN})$ is simulated using our particle-based simulator and the results plotted for various values of radius and reaction probability of obstacle. The simulation results for existence of an obstacle are compared with some analytical expressions with absence of the interfering obstacle for both passive and fully absorptive receivers. This obstacle has a significant impact on the received signal (concentration profiles) or the reliability of the communication links between nanomachines. 
This paper is organized as follows. Firstly, we introduce the system model and theoretical aspects of molecular diffusion, passive receiver, and absorptive receiver in section II. In section III, the analytical and simulation results are plotted and discussed. Finally, the paper is concluded in section IV.

\section{SYSTEM MODEL}

Here we propose a model for the molecular communication via diffusion (MCvD) and present the channel (medium) impulse responses for passive and absorptive receivers under normal diffusion. We assume there is a reactive obstacle placed between the transmitting nanomachine $(\mathrm{TN})$ and receiving nanomachine $(\mathrm{RN})$ in an unbounded molecular fluidic environment which hinders the movement of the molecules as illustrated in Fig. 1. The TN is assumed to be a point-like source which encodes the information on the molecular concentration, known as concentration shift keying (CSK), before emitting the molecules instantaneously into the propagating medium (channel). The size of TN is assumed to be negligible compared with the relative distance between $\mathrm{TN}$ and $\mathrm{RN}$.

(a)

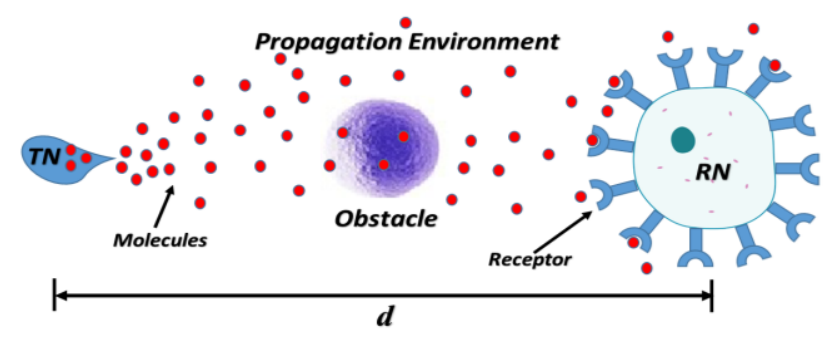

(b)

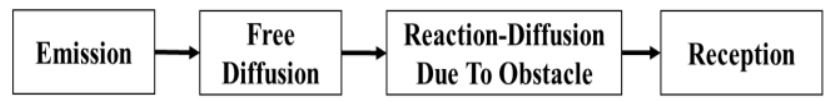

Fig. 1. Molecular communication in a diffusive medium (channel) with reactive obstacle. (a) Graphical representation. (b) Block diagram representation.

Once released, the information molecules diffuse randomly in all directions in the environment according to Brownian motion. The channel could be a biological environment such as blood vessels and we assume that the collisions of the molecules with the boundary are elastic (i.e., unbounded environment). The molecules will collide and react with a partially absorptive obstacle located at the middle point between TN and RN. This obstacle will absorb some of the information molecules and some of them will reflect back before they reach the $\mathrm{RN}$ depending on the reaction probability of the obstacle. At the target site, the molecules which success in reaching the RN will be either counted without absorbing for passive $\mathrm{RN}$ or they will be absorbed and removed from the propagation environment for fully absorptive RN. The number of the received molecules over time at the $\mathrm{RN}$ represents the received signal.
Fick's second law of diffusion [7] characterizes mathematically the diffusion of molecules in a fluidic environment as follows [11]

$$
\frac{\partial h(x, y, z, t)}{\partial t}=D \nabla^{2} h(x, y, z, t)
$$

where $\nabla^{2}$ is the Laplacian operator and $h(x, y, z, t)$ is the spatiotemporal distribution of information molecules at the point $(x, y, z)$ and at the time $t$.

The diffusion coefficient $(D)$ of the environment is given by [10] as

$$
D=\frac{K_{B} T_{a}}{6 \pi \eta r_{m}}
$$

where $K_{B}$ is the Boltzmann constant, $T_{a}$ is the absolute temperature in (Kelvin), $\eta$ is the dynamic viscosity of the fluid environment, and $r_{m}$ is the Stoke's radius of the information molecules. In this work, we assume that the diffusion coefficient is constant and uniform in all the directions (i.e., isotropic diffusion).

Existence of a passive $\mathrm{RN}$ in the environment will not affect the diffusion of the released molecules; therefore, they can enter throughout the passive RN boundary without any physical or chemical reactions. Thus, the channel impulse response at any arbitrary location $(x, y, z)$ due to a TN located at coordinate $\left(x_{0}, y_{0}, z_{0}\right)$ considering the three dimensional domain of the channel can be written as [12]

$$
h(x, y, z, t)=\frac{1}{(4 \pi D t)^{3 / 2}} \exp \left(-\frac{d^{2}}{4 D t}\right), \quad t \geq 0
$$

where $\mathrm{d}$ is the separation distance between $\mathrm{TN}$ and $\mathrm{RN}$ and thus $d^{2}=\left(x-x_{0}\right)^{2}+\left(y-y_{0}\right)^{2}+\left(z-z_{0}\right)^{2}$.

Equation (3) represents the solution of the diffusion equation (1) with an initial condition of the impulsive emission of molecules and boundary condition of an elastic collisions of molecules with the boundary (i.e. unbounded medium).

In general, the total received molecules at a passive $\mathrm{RN}$ can be calculated by integration of channel impulse response over the volume of $\mathrm{RN}$ then multiplying the result by the initial strength of the source (i.e., the total number of released molecules at time $t=0$ ). Under the assumption that the distance between $\mathrm{TN}$ and $\mathrm{RN}$ is large compared to the receiver size, then the concentration inside the receiver volume is uniformly distributed and thus the number of received molecules by a passive $\mathrm{RN}$ can be expressed as [11]

$$
N_{P}=\alpha V_{r x} h(x, y, z, t)
$$

where, $\alpha$ is the total number of released molecules at a time $t=0, V_{r x}$ is the volume of a spherical $\mathrm{RN}$ given as $V_{r x}=4 \pi / 3 R_{r x}^{3}$, and $R_{r x}$ is the receiver radius. 
The signal peak time is the time at which the signal has the maximum amplitude. The peak time for the molecular received signal by a passive $\mathrm{RN}$, i.e., Eq. (4), can be obtained by finding the time instant at which the time derivative of (3) vanishes. Then, after substituting this peak time value in (4), we get an expression for the peak amplitude which derived in [12] as

$$
N_{\max }=\frac{\alpha}{d^{3}}\left(\frac{3}{2 \pi e}\right)^{3 / 2}
$$

The information molecules react with protein receptors on the surface of the RN once they hit its boundary and thus they may be absorbed or reflected by the RN. This means that the information molecules contribute to the signal once in a short duration. Therefore, existence of an absorptive RN will affect the channel impulse response.

For fully absorptive RN in unbounded environment, the accumulative number of received molecules until time $t$ is given by [14] as

$$
N_{A}=\alpha \frac{R_{r x}}{d} \operatorname{erfc}\left(\frac{d-R_{r x}}{\sqrt{4 D t}}\right)
$$

where, $\operatorname{erfc}($.$) is the complementary error function.$

\section{RESULTS}

In this section, we examine the impact of a reactive (absorptive) obstacle, located between $\mathrm{TN}$ and $\mathrm{RN}$, on the molecular received signal for both passive and absorptive RN via reaction-diffusion particle-based simulation. The radius and reaction probability of the obstacle are varied to examine their impact on the number of the received molecules by the RN. Then, these results are compared with the analytical results of the case of absence of the obstacle. The simulation parameters are listed in table I unless state otherwise.

In the particle-based simulator, the total simulation time $T$ is divided into $N$ time steps of width $\Delta t$. The information molecules are released by a point-like TN at the time $t=0$ and at the location $\left(x_{0}, y_{0}, z_{0}\right)$. Then, the information molecules move randomly and independently of each other according to Brownian motion in all direction in unbounded environment. The precise position of each molecule in the environment is tracked and updated at each time step as follow [3]

$$
\left(x_{i}, y_{i}, z_{i}\right)=\left(x_{i-1}, y_{i-1}, z_{i-1}\right)+\left(\Delta x_{i}, \Delta y_{i}, \Delta z_{i}\right), \quad i=1, \ldots, N
$$

Where $N$ is the total number of the simulation time steps, $\left(x_{i}, y_{i}, z_{i}\right)$ is the new position of the molecule at the $i^{t h}$ time step, $\left(x_{i-1}, y_{i-1}, z_{i-1}\right)$ is the previous position of the molecule at the $(i-1)^{t h}$ time step, and $\left(\Delta x_{i}, \Delta y_{i}, \Delta z_{i}\right)$ is the random displacements over each coordinate at $i^{\text {th }}$ time step which follows the normal distribution $N\left(0, \sigma^{2}\right)$ with zero-mean and variance $\sigma^{2}=2 D \Delta t$.
Each information molecule hit the obstacle will assign with a random number uniformly distributed between zero and one. Then, if the assigned number is smaller than the reaction probability, the molecule will be absorbed by the obstacle and will remove from the simulation environment. Otherwise, the molecule will reflect-back to the environment.

TABLE I. SIMULATION PARAMETERS.

\begin{tabular}{c|c}
\hline Parameter & Value \\
\hline Simulation time $(T)$ & $1-5 \mathrm{~s}$ \\
\hline Time step $(\Delta t)$ & $1 \mathrm{~ms}$ \\
\hline No. of emitted molecules $(\alpha)$ & 10,000 \\
\hline Diffusion coefficient $(D)$ & $100 \mu \mathrm{m}^{2}$ \\
\hline Radius of RN $\left(R_{r x}\right)$ & $2 \mu \mathrm{m}$ \\
\hline Radius of obstacle $\left(R_{o b s}\right)$ & $2 \mu \mathrm{m}$ \\
\hline Distance between TN and RN & $10 \mu \mathrm{m}^{2}$ \\
\hline Reaction probability of obstacle $(P)$ & $\{0,0.5,1\}$ \\
\hline Number of iterations & 200 \\
\hline
\end{tabular}

Fig. 2 shows the number of received molecules as a function of time for a passive receiver with and without existing of the obstacle between $\mathrm{TN}$ and $\mathrm{RN}$ for different reaction probability at the obstacle. In the case of absence the obstacle, the number of received molecules has a higher value compared with existence of the obstacle. Moreover, as the reaction probability for the obstacle increases, the amplitude of received signal decreases. This because that some of the molecules will be absorbed by the obstacle and will not reach the receiver in orders to contribute to the received signal. The chance for molecules to be absorbed by the obstacle increases as the reaction probability increases and thus the number of the received molecule at the receiver will be reduced.

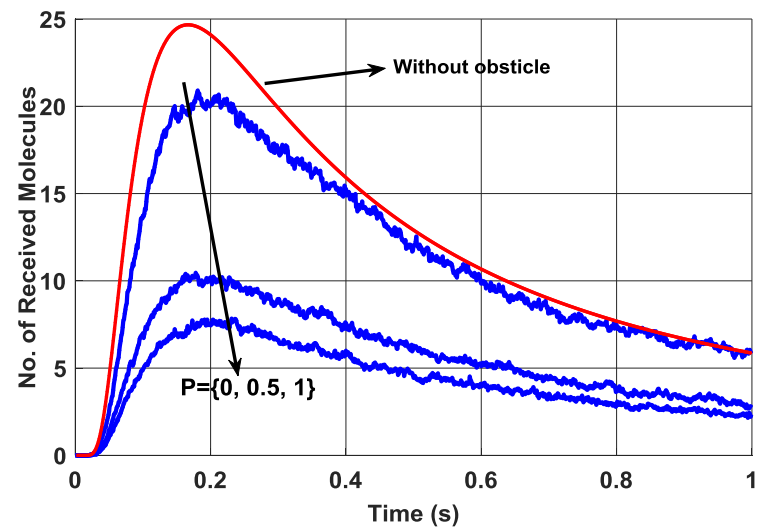

Fig. 2. The number of received molecules for a passive receiver for various values of the reaction probability of obstacle.

The peak amplitude of the received signal as a function of obstacle's radius for a passive receiver is shown in Fig. 3 for different values of the reaction probability of the obstacle. As shown in this figure, the peak amplitude of the received signal decreases with increasing of the obstacle's size. As the radius of the obstacle increases, the chance of absorbing of 
the molecules by the obstacle increases and thus fewer numbers of the molecules can succeed in reaching the receiver. However, in the case of absence of the obstacle, the peak amplitude has a higher value than the case of existence of the obstacle for all the values of the reaction probability.

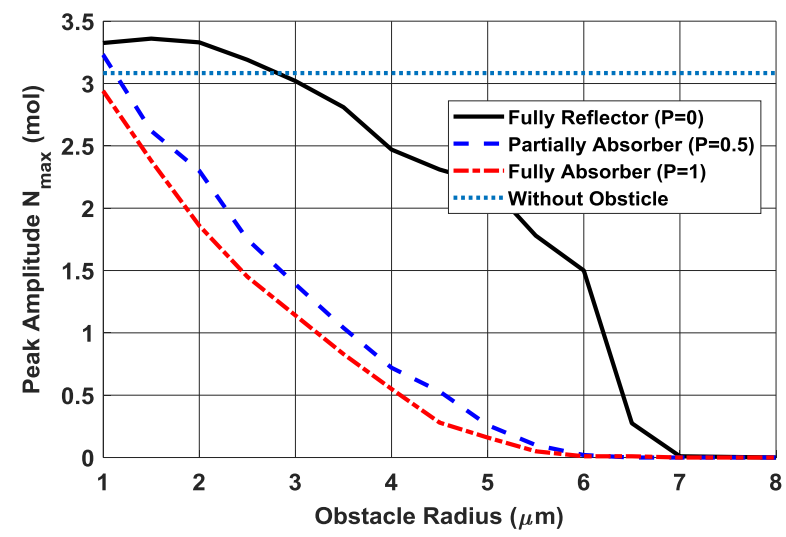

Fig. 3. The peak amplitude for a passive receiver as a function of obstacle radius for various values of the reaction probability of obstacle for $20 \mu \mathrm{m}$ separation distance between $\mathrm{TN}$ and $\mathrm{RN}$.

Fig. 4 shows the cumulative number of the received molecules with time at a full absorptive receiver with/without existence of the obstacle and for different reaction probabilities. As expected, the absence of the obstacle gives the higher amplitude of the received signal compared with the case of existence of the obstacle between TN and RN. Furthermore, the higher reaction probability of the obstacle leads to the lower amplitude of the received signal. At the very large time, the received signal reaches the steady state and becomes fixed with time.

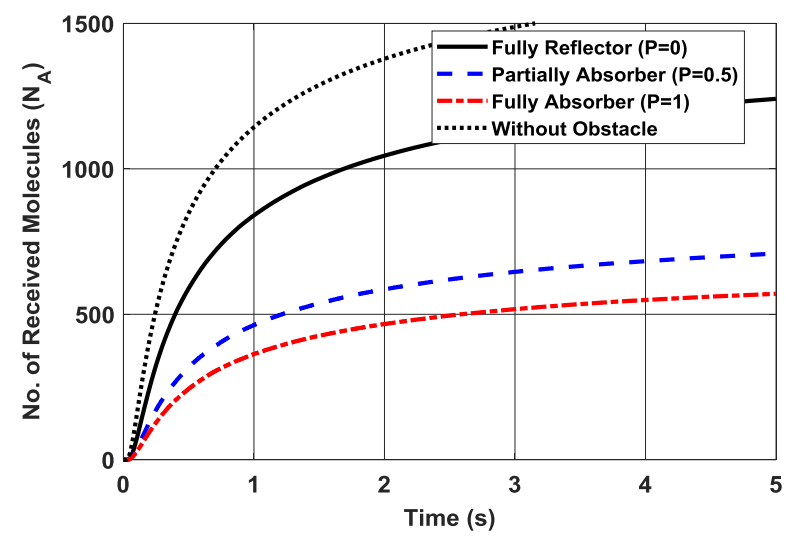

Fig. 4. The expected number of received molecules for fully absorptive receiver as a function of time for various values of the reaction probability of obstacle.

\section{CONCLUSIONS}

In this paper, we examine the impact of a reactive obstacle, located between the transmitting nanomachine (TN) and the receiving nanomachine $(\mathrm{RN})$, on the molecular received signal. We develop a particle-based simulator to predict the number of the received molecules at the $\mathrm{RN}$ for both passive and absorptive receivers. The impacts of radius and reaction probability of obstacle on the received signal are examined. Moreover, we compare the effect of the obstacle on the received signal with the case of absence of the obstacle.
The results show that the obstacle has a significant impact on the received signal (concentration profiles) and thus on the reliability of the communication links between the nanomachines. The amplitude of the received signal decreases in the case of existence of the obstacle between $\mathrm{TN}$ and $\mathrm{RN}$ for both the passive and absorptive receivers. This reduction increases as the reaction probability and size of the obstacle increases.

Thus, it is important to consider the impact of the reactive obstacles. e.g., cells, between the transmitter and the receiver in order to accurately predict the molecular received signal at the target site.

\section{REFERENCES}

[1] I. F. Akyildiz, F. Brunetti, and C. Blázquez, "Nanonetworks: A new communication paradigm," Computer Networks, vol. 52, pp. 2260$2279,2008$.

[2] T. Nakano, A. W. Eckford, and T. Haraguchi, Molecular communication: Cambridge University Press, 2013.

[3] N. Farsad, H. B. Yilmaz, A. Eckford, C.-B. Chae, and W. Guo, "A comprehensive survey of recent advancements in molecular communication," IEEE Communications Surveys \& Tutorials, vol. 18, pp. 1887-1919, 2016.

[4] P.-C. Yeh, K.-C. Chen, Y.-C. Lee, L.-S. Meng, P.-J. Shih, P.-Y. Ko, et al., "A new frontier of wireless communication theory: diffusionbased molecular communications," IEEE Wireless Communications, vol. 19, 2012

[5] T. Nakano, M. J. Moore, F. Wei, A. V. Vasilakos, and J. Shuai, "Molecular communication and networking: Opportunities and challenges," IEEE transactions on nanobioscience, vol. 11, pp. 135$148,2012$.

[6] H. C. Berg, Random walks in biology: Princeton University Press, 1993.

[7] J. Crank, The mathematics of diffusion: Oxford university press, 1979.

[8] M. Pierobon and I. F. Akyildiz, "A physical end-to-end model for molecular communication in nanonetworks," IEEE Journal on Selected Areas in Communications, vol. 28, 2010.

[9] M. Ş. Kuran, H. B. Yilmaz, T. Tugcu, and B. Özerman, "Energy model for communication via diffusion in nanonetworks," Nano Communication Networks, vol. 1, pp. 86-95, 2010.

[10] A. Noel, K. C. Cheung, and R. Schober, "Optimal receiver design for diffusive molecular communication with the flow and additive noise," IEEE transactions on nanobioscience, vol. 13, pp. 350-362, 2014.

[11] D. Kilinc and O. B. Akan, "Receiver design for molecular communication," IEEE Journal on Selected Areas in Communications, vol. 31, pp. 705-714, 2013.

[12] I. Llatser, A. Cabellos-Aparicio, M. Pierobon, and E. Alarcón, "Detection techniques for diffusion-based molecular communication," IEEE Journal on Selected Areas in Communications, vol. 31, pp. 726734, 2013.

[13] A. Aijaz and A.-H. Aghvami, "Error performance of diffusion-based molecular communication using pulse-based modulation," IEEE transactions on nanobioscience, vol. 14, pp. 146-151, 2015.

[14] H. B. Yilmaz, A. C. Heren, T. Tugcu, and C.-B. Chae, "Threedimensional channel characteristics for molecular communications with an absorbing receiver," IEEE Communications Letters, vol. 18, pp. 929-932, 2014.

[15] M. ELKASHLAN, "Modeling and simulation of molecular communication systems with a reversible adsorption receiver," 2016.

[16] Y. Lu, M. D. Higgins, A. Noel, M. S. Leeson, and Y. Chen, "The effect of two receivers on broadcast molecular communication systems," IEEE transactions on nanobioscience, vol. 15, pp. 891-900, 2016. 